\title{
Understanding Social Complex Systems with PlatBox Simulator
}

\author{
Takashi Iba *1 \\ Nozomu Aoyama*2 \\ *1 Faculty of Policy Management, Keio University \\ *2 SFC Institute, Keio University
}

\begin{abstract}
This paper presents a framework and tools for modeling and simulating societies as complex systems. Although the concept of the complex systems has been highly demanded in social sciences, there is no satisfactory scheme for modeling and simulating it. In this paper, we propose the model framework, which we call "PlatBox Foundation Model", and tools, which we call "PlatBox Simulator" and "Component Builder". Then we take an example of word-of-mouth simulation based on the framework and tools, and discuss the difference from the existing systems.
\end{abstract}

Keywords: Complex Systems, Computer Simulation, Multi-Agent-Based Model

\section{Introduction}

This paper presents a framework and tools for modeling and simulating societies as complex systems. Although the concept of the complex systems has been highly demanded in social sciences, there is no satisfactory scheme for modeling and simulating it. Many researchers and we almost agree that the multi-agent-based model is suitable for studying complex systems, however in the current state, there is a problem that needs to be resolved. The problem is the absence of integrated environment to support a whole research process, from conceptual modeling to simulation analysis. The problem did not become serious too much up to now, because the models were small-scale and for experimental use. It becomes, however, indispensable to resolve the problems, as the simulations come to be used practically in social science and policy analysis.

In this paper, we introduce an object-oriented computational modeling for social sciences in order to model and simulate complex systems where the model framework "PlatBox Foundation Model" is proposed. Moreover, in order to support modeling based on the framework, "Component Builder" (CB) is proposed. In addition, "PlatBox Simulator" is proposed for simulating and analyz-

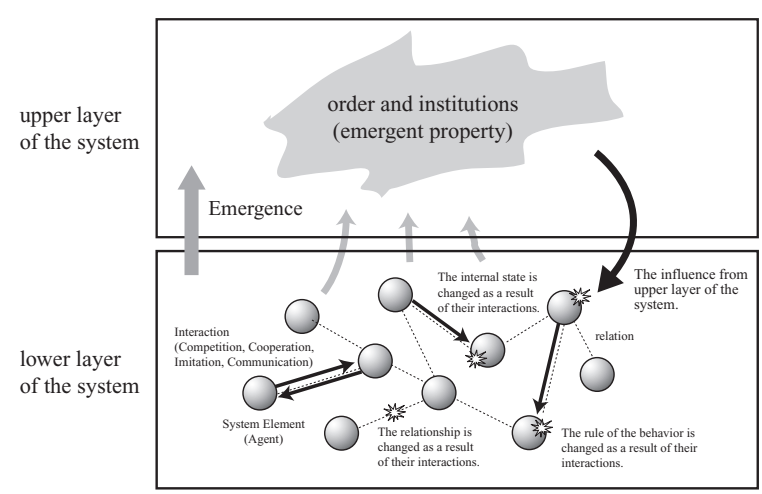

Figure 1: An overview of complex systems

ing the models ${ }^{1}$.

\section{Complex Systems}

We will begin at considering the nature of our targets. There is no shared definition of complex systems among the scientists, but we can say that the definition is able to be summarized in two ways as follows. In a broad sense, the complex system means that the system has the components where each component changes the internal states by mutually interacting with the other components. In addition, in a strict sense, the complex system means the system where the rules of each component's behavior are changed dynamically during the simulation. These changes often occur under the influence of the macroscopic situation that was emerged from microscopic interactions (Figure 1). In this paper, we propose the framework and tools for modeling and simulating the complex systems as defined above.

\footnotetext{
${ }^{1}$ The framework was originally proposed as the name of "Boxed Economy Foundation Model"[1]. And the simulator is a succeeding version of "Boxed Economy Simulation Platform" (BESP). Although we've worked toward development of framework and tools for modeling and economic society since 1999 [2], we were getting to think that the use of our framework is not restricted to the economic field. For this reason, we changed the project name to "PlatBox Project", the name of framework to "PlatBox Foundation Model", and the name of software to "PlatBox Simulator" in 2005 .
} 


\section{Model Framework}

We would like to propose the model framework, "PlatBox Foundation Model" (Figure 2), in order to introduce an object-oriented computational modeling for social sciences for modeling and simulating complex systems.

\subsection{Role of the framework}

PlatBox Foundation Model is the model framework which defines the set of concepts for modeling societies, and which supports from the analysis of target world to the execution of simulations.

In the viewpoint of conceptual modeling, PlatBox Foundation Model provides, as a model framework, (1) a frame of reference for recognizing the target world, (2) a vocabulary for describing the concepts obtained by recognition, and (3) a code for communications among the modelers. And, in the viewpoint of simulation design, PlatBox Foundation Model provides, as a software framework, (1) transformation rules from conceptual models to simulation models, (2) supports for implementing the simulation models, and (3) architecture for sharing and reusing simulation models.

\subsection{Major classes of the framework}

The framework defines the set of concepts for modeling societies, consisting five major elements: "Agent", "Relation", "Behavior", "Goods", and "Information". "Agent" object is defined to describe an autonomous actor who does an action. The relation between agents will be described by "Relation" object. The behavior of the agent is defined as "Behavior" object, which is described as a state machine that is a system which changes the state when the event is received. "Goods" can be defined as material / immaterial things which are possessed by Agents in order to be used or to be exchanged with other agents. The information which is possessed by Goods or Agents is defined as "Information" in the model.

\section{Modeling Tools}

In order to support making the simulation models with the proposed framework, we propose tools for modeling and simulating social complex systems: "PlatBox Simulator" and "Component Builder". PlatBox Simulator is a software platform to execute and to analyze the agent-based social simulations. It is developed to realize an extensible software application with the component-based architecture. The modeler can obtain the simulation

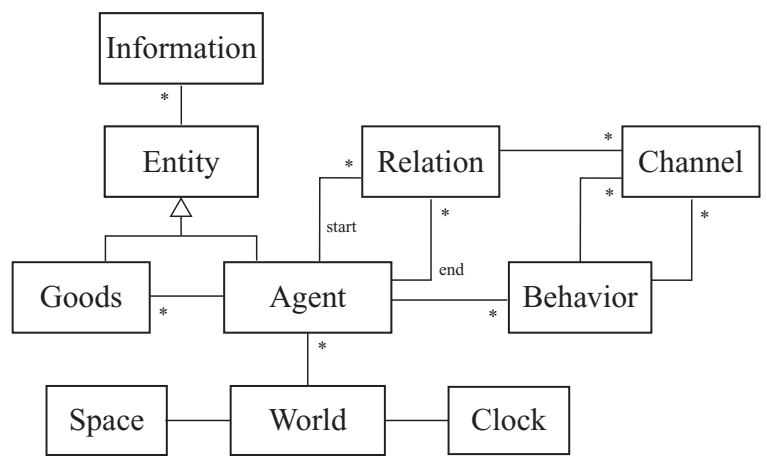

Figure 2: Major classes of PlatBox Foundation Model

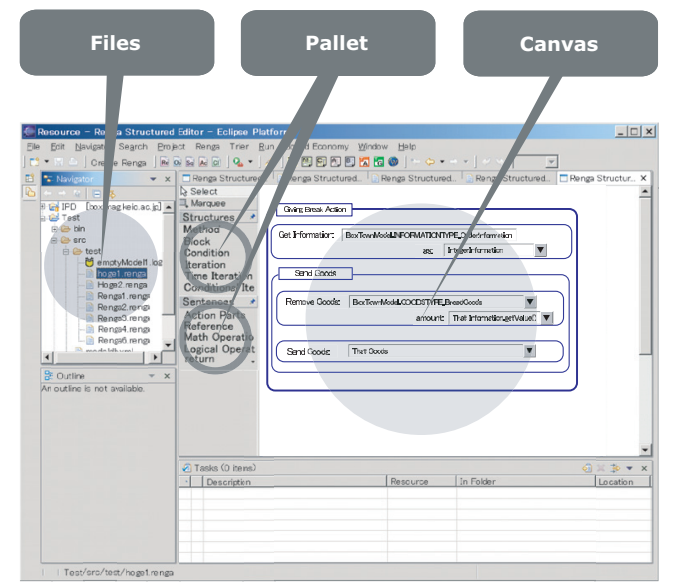

Figure 3: Action Designer of Component Builder

environment which suits the needs, only if he/she sets necessary model components into the platform.

Component Builder is the tool for designing the model component plugged into PlatBox Simulator. With using the tool, a modeler can develop a simulation program just by drawing model diagrams in the modeling language. As a result, the modeler comes to be able to make the simulation as long as they have little skills of programming. Moreover, the modeler can make and change their model promptly, and then can give priority to the trial and error in modeling and the analysis of the consequences.

Component Builder consists of five designers and a composer: "Model Designer" for modeling the static view of the simulation, "Behavior Designer" for modeling the behavior of agents, "Action Designer" for describing details of actions of behavior (Figure 3), "Activity Designer" and "Communication Designer" for conceptual modeling, and "World Composer" for setting the initial state of the simulation world. They are the tools 
to generate the program code just by making the diagram and setting the parameters with a graphical user interface [3].

\section{An Example}

As an example of a simulation model built with Component Builder and PlatBox Simulator, we introduce "Movie Theater Model" here.

\subsection{Model Overview}

The movie theater we focused is independent theater that shows films like art films instead of major films. Due to its small market and resource for advertising, word-of-mouth marketing becomes essential to the theaters. Therefore, the purpose of simulating and analyzing "Movie Theater Model" is to find the most efficient method of advertising using word-of-mouth marketing.

\subsection{Model Description}

In this model, there are two agents: "Movie Theater" and "Customer" (Figure 4). Movie Theater agent has "Advertising Behavior", and Customer agent has "Chatting Behavior". The relation from Movie Theater agent to Customer agent is "Advertising Target Relation", and the relation among the Customer agents is "Friend Relation". The information which these agents send and memorize is "Movie Information".

Based on these elements, the simulation steps are as follows. First, Movie Theater agent randomly selects a numbers of Customer agents, and then links an Advertising Target Relation. Second, Movie Theater agent makes Movie Information, Third, Movie Theater agent sends Movie Information to selected Customer agents. Forth, Each Customer agent sends Movie Information to Customer agents with Friend Relation. Then, repeat Step.4. In modeling on Component Builder, we draw the statechart diagram and action block diagram in order to describe the flow of the simulation. There is no space to show all diagrams in this paper, so we take only two diagrams as examples (Figure 5, 6).

\subsection{Code Generation}

After generating the program code from the diagrams, the simulation will be executed on PlatBox Simulator (Figure 7). We can observe the process of information becoming widespread, changing the initial settings. In this case, we draw 5 diagrams, which are a class diagram, two statechart diagrams, and two action block diagrams,

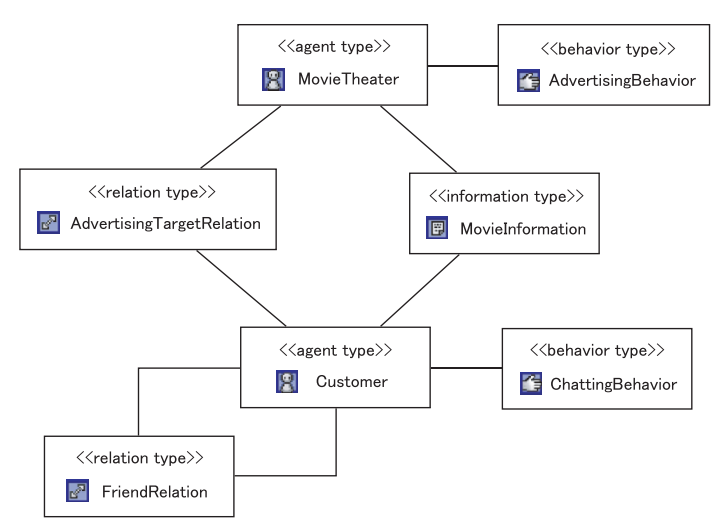

Figure 4: Class diagram (Movie Theater Model)

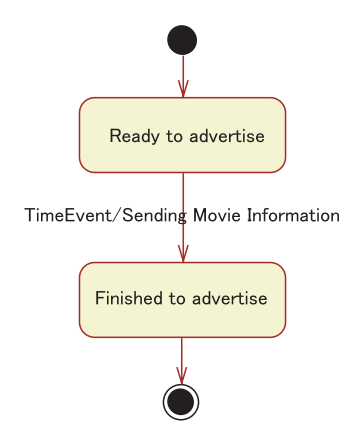

Figure 5: Statechart diagram of Advertising Behavior (Movie Theater Model)

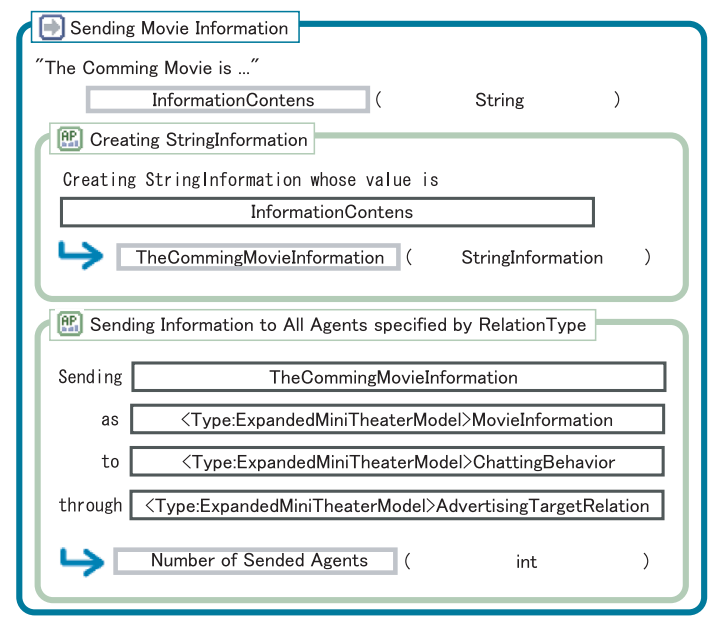

Figure 6: Action block diagram of a part of action in Advertising Behavior (Movie Theater Model) 


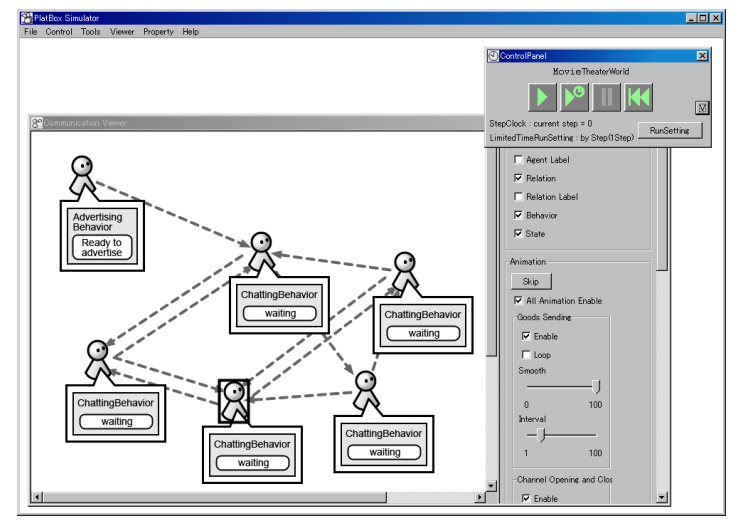

Figure 7: Simulating Movie Theater Model on PlatBox Simulator

and set 1 world-setting. From the diagrams and settings, Component Builder generate 1043 lines program. The breakdown of code size is shown in Table 1.

\section{Discussion}

In the final place, we shall make clear where our framework and tools are different from the existing systems. In the last some years, several languages, frameworks and tools for agent-based simulations have been proposed: "Swarm Simulation System"[4], "RePast"[5], "Ascape" [6], and "LSD" [7]. These support systems assist the modelers to write programs by providing a general library and framework, and in fact these systems are useful for the reduction of programming. These systems, however, would not support for the modelers to do modeling. On the contrary, our approach, which providing a model framework, process, and tools, is to support a whole process from conceptual modeling to simulation implementation.

From the viewpoint of software engineering, our approach is based on the emerging development process, which is driven by modeling: "ModelDriven Development" [8]. We can use the highlevel language for development, instead of writing

Table 1: Amount of the diagrams and program code of Movie Theater Model

\begin{tabular}{l|l|l}
\hline Tool & Diagram & generated code \\
\hline \hline Model & 1 diagram & 82 lines \\
\hline Behavior & 2 diagram & 184 lines \\
\hline Action & 2 diagram & 305 lines \\
\hline World & 1 setting & 472 lines \\
\hline
\end{tabular}

in the lower-level language (program code). In this sense, design models are development artifacts as same as design artifacts, therefore the design model is no longer "just paper" and "blueprint". Therefore, the design models are development artifacts that contribute directly to simulation development.

\section{Conclusion}

In this paper, we proposed a framework and tools for modeling and simulating societies as complex systems. We have already applied the proposed framework and tools to several social phenomena, for instance, financial market, business competition, social network, evolutionary game theory, and queuing model. Our experiences show that our proposed framework and tools are quite powerful and have the potential for supporting thinking and communicating about complex societies and economies. The tools proposed in this paper are open to the public on http://www.platbox.org/. And please contact us, if you are interested in our challenge.

\section{References}

[1] Iba, T.: A Framework and Tools for Modeling and Simulating Societies as Evolutionary Complex Systems. 2nd. International Conference of the European Social Simulation Association. (2004)

[2] Iba, T., Hirokane, M., Takabe, Y., Takenaka, H., Takefuji, Y.: Boxed Economy Model: Fundamental Concepts and Perspectives. Proceedings of Computational Intelligence in Economics and Finance. United States (2000)

[3] Nozomu Aoyama, Rintaro Takeda, Takashi Iba and Hajime Ohiwa.: Simulation Development Tools with MDA. International Workshop on Massively Multiagent Systems (2004)

[4] Luna, F., and Stefasson, B. (eds.): Economic Simulations in Swarm: Agent-Based Modelling and Object Oriented Programming. Kluwer Academic Publishers (2000)

[5] Collier, N.: RePast: An Extensible Framework for Agent Simulation. http://repast. sourceforge.net/ (2003)

[6] Parker, M. T.: What is Ascape and Why Should You Care?. Journal of Artificial Societies and Social Simulation. vol. 4, no. 1.

[7] Andersen, E. S., Valente, M.: The art of simulation and the Lsd system. http://www.busi ness.aau.dk/evolution/ (2003)

[8] Iba, T., Matsuzawa, Y., and Aoyama, N.: From Conceptual Models to Simulation Models: Model Driven Development of Agent-Based Simulations. 9th Workshop on Economics and Heterogeneous Interacting Agents. Kyoto, Japan (2004) 